\title{
Angle of Twist of a Double-Bent Leaf Flexure under Torsion
}

\author{
Nghia Huu Nguyen ${ }^{1,2}$ - Hong-Cheol Lee ${ }^{1}$ - Sunghee Jo ${ }^{1}$ - Dong-Yeon Lee ${ }^{1, *}$ \\ 1Yeungnam University, Department of Robotics and Intelligent Machine, Republic of Korea \\ ${ }^{2}$ Nha Trang University, Faculty of Mechanical Engineering, Viet Nam
}

\begin{abstract}
This paper describes the rotational displacement of the double-bent leaf flexure subjected to a torsional load. Warping and specifically double partially-restrained warping are considered when the torsion is applied. The warping restraint factor for partially-restrained warping is also considered. Castigliano's theorem is applied to derive the theoretical equations of the angle of twist. The results for the fully restrained, partially restrained, and free warping cases are compared and verified with finite element analysis (FEA). A sensitivity analysis over the length, width, and thickness is performed and the results are compared with FEA. The errors between the theory result with the warping restraint factor $(K=0.5)$ and those of FEA are the lowest, below 5\%. This suggests that the angle of twist of the double-bent leaf flexure under torsion is accurately predicted by considering double partially-restrained warping.

Keywords: torsion, double-bent leaf flexure, warping restraint factor, partially restrained warping, Castigliano's theorem, finite element analysis

Highlights
- The warping and double partially-restrained warping are considered when torsion is applied to the double-bent leaf flexure.
- The theoretical equations of the angle of twist of double-bent leaf flexure are derived by applying Castigliano's theorem.
- The theoretical results are compared and verified with finite element analysis; errors are below 5\%.
\end{abstract}

\section{INTRODUCTION}

Leaf springs and hinges are two kinds of flexure guide; they have many applications in precision machines, especially in nanoelectromechanical systems (NEMs) and microelectromechanical systems (MEMs). These flexure guides have outstanding advantages such as a lack of friction, and their guiding resolution is on the order of a nanometre. However, they have a short working range (from micrometre to millimetre). Therefore, the new flexure guides, which have a large working range and nano-resolution, have become very important in recent years. For example, a parallel leaf spring flexure has been presented with increasing displacement [1]; a displacement reduction mechanism based on torsional leaf spring hinges has been developed [2]; a novel flexure-based leaf spring has been presented with a large range $(25 \times 25) \mu \mathrm{m}^{2}$ [3]; a mechanism for a single-axis flexure-based nano-positioning stage with a large range of motion (up to a millimetre) is described in [4]; a multi-axis positioning system with nanometric resolution over a $1 \mathrm{~mm}$ stroke is described in [5]; a novel flexure parallel-kinematics precision positioning stage with a centimetre range has been designed and developed [6], and many hinge flexure guides have been reported [7] to [11]. In comparison with a hinge, the leaf spring has a smaller size and a minimum use of materials for the same displacement. Thus, we examined leaf spring flexure guides for the large range travel in this study.
In general, a leaf spring can be divided into three types: single, single-bent, and double-bent leaf [12]. The single leaf has two degrees of freedom (DoF) with a coupled error and a small range of travel. Singlebent and double-bent leaf flexures (DBLFs) have three DoF and a larger range of travel. Many studies in recent years have focused on using leaf springs in precision guided machines [1], [2] and [12] to [16]. The bending deflections and stresses of a cantilevered single-leaf flexure using higher-order beam theory were investigated [13]; a one DoF rotational flexure joint using a leaf spring has been proposed [14]; the bending displacement of a single-bent leaf flexure under transverse load has been analysed [15], and a leaf spring has been designed in combination with a hinge [2] and [16]. Even though the double-bent leaf flexure can create a larger range of travel than a single-bent leaf flexure, much research has not been reported. Thus, the DBLF under torsional loading will be investigated in this study. The U-spring model presented in [17] has a model similar to the DBLF, but only the in-plane deformations were analysed and a torsional analysis was not considered in the compliances of their model [17].

Torsional analysis plays an important role in machine design, especially in precision machines. General torsional theory considers the cases of no warping and warping in torsion was shown in [18] to [20]. Torsional theory with warping has been presented in many studies. Examples include a generalized 
beam theory of bars under torsional loading presented in [21], and a theory of torsional analysis for an open thin-walled beam developed in [22] and [23]. However, the case of partially restrained warping was not considered in these studies. The partially restrained warping case was analysed and presented in [24] and [25]; however, it was applied to a different analysis model. The torsional analysis of a leaf spring with partially-restrained warping was investigated in [26], but it was applied to a single-bent leaf flexure in which the partially restrained warping occurred one time.

In the present study, we performed a torsional analysis of a DBLF with consideration of double partially-restrained warping and changes of the warping restraint factor $K$. The strain energy of each element of the DBLF was derived, and the theoretical torsional displacement equation of the DBLF was derived using Castigliano's second theorem. The angle of twists of the DBLF under torsional loading from the theoretical equations is numerically compared with those from a finite element analysis (FEA) at the default values and with variations of the length, width, and thickness of the DBLF. We suggest that our torsional analysis could be applied to applications of DBLFs in nano-scanner design.

\section{THEORETICAL ANALYSIS}

A model of the DBLF is shown in Fig. 1. The DBLF was divided into three elements. It has one fixed end (at the free end of element 3), and three forces and three moments are applied to the other end. In this structure, the flexure acts as a leaf spring with three degrees of freedom and can create a large range of travel. The dimensions of the structure are length $l$, width $b$, and thickness $t$. When a torsional moment $T_{x}$ is applied at the free end of element 1 , element 1 twists, element 2 bends, and element 3 twists. The joints between elements 1 and 2 and elements 2 and 3 are not fixed and are not free; they produce double partially-restrained warping under torsional loading.

Fig. 2 shows an application of the DBLF in a planar nano-scanner. The system includes four flexures connected to four corners of a square moving body in order to provide three DoF motion smoothly and has a larger range of travel than other leaf spring types. When a torsion along the $x$ or $y$ axis is externally applied in the moving body, it causes out-of-plane motion: the moving body cannot retain a planar surface, which will lead to reduced scanner accuracy. In this investigation, the rotational displacement of the flexure under torsion $T_{x}$ was analysed and calculated to ensure accurate operation of the precision machine.

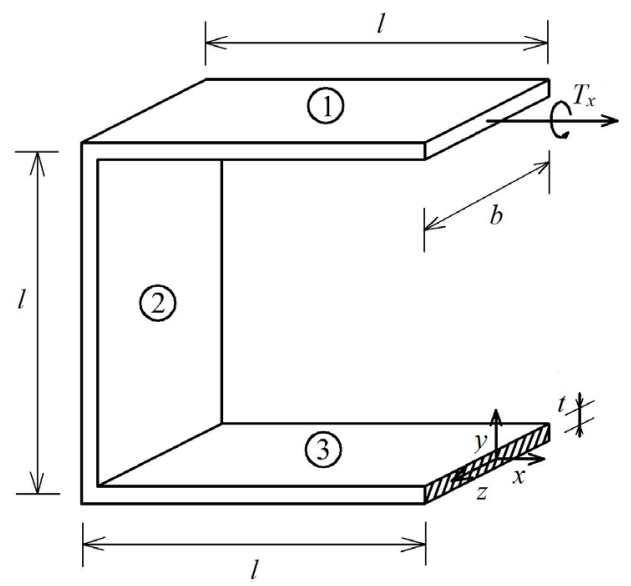

Fig. 1. Schematic diagram of the double-bent leaf flexure

Double-bent leaf flexure

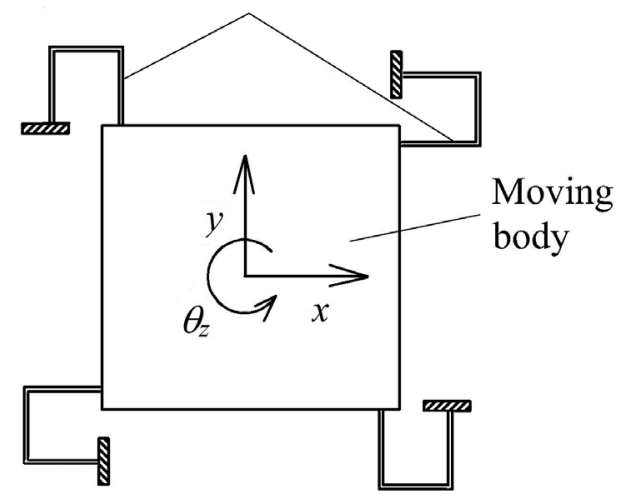

Fig. 2. Schematic diagram of a planar scanner

\subsection{Derivation of Total Strain Energy}

We used Castigliano's theorem to find the rotational displacement of the flexure shown in Fig. 1. The twist angle was defined by using the partial derivative of the total strain energy with respect to the applied torsion $T_{x}$ as follows:

$$
\theta_{x}=\frac{\partial U}{\partial T_{x}}
$$

where $U$ is the total strain energy that is stored in the flexure and $\theta_{x}$ is the angle of twist.

When the torsion moment $T_{x}$ is applied to the flexure, it causes torque in element 1 , bending in element 2, and torque in element 3. Thus, three components of strain energy due to $T_{x}$ must be defined. The governing equation for non-uniform torsion of a 
homogeneous isotropic prismatic bar subjected to an end constant torsion $T_{x}$ is given by [19]

$$
G J_{x} \frac{d \theta_{x}}{d x}-E C_{w} \frac{d^{3} \theta_{x}}{d x^{3}}=T_{x},
$$

where $J_{x}, C_{w}, G$, and $E$ are the torsion constant, warping constant, modulus of transverse elasticity, and modulus of longitudinal elasticity (Young's modulus), respectively. The general solution of Eq. (2) is given by [19]:

$$
\theta_{x}=C_{1}+C_{2} x+C_{3} \cosh \alpha x+C_{4} \sinh \alpha x,
$$

where $\alpha=\sqrt{\frac{G J_{x}}{E C_{w}}}$ is the torsion-bending constant and $C_{k}(k=1,2,3,4)$ are the constants of integration. The angle of twist is defined after the boundary conditions (BCs) are applied.

\subsubsection{Strain Energy of Torsion in Element 1}

As previously mentioned, the joint between element 1 and 2 of the DBLF is partially restrained from warping. Therefore, the partial warping constraint occurs at $x=-l$ and free warping occurs at $x=0$. To find the angle of twist of element 1 with the partial warping constraint at the joint, the following two extreme BCs are defined and combined as follows:

First case. Given the assumptions that the full warping constraint occurs at $x=-l$ and the free constraint occurs at $x=0$, we have $\theta_{x}(-l)=0$, $\frac{d \theta_{x}(-l)}{d x}=0$ at $x=-l$; and $M_{\omega}=0$ or $\frac{d^{2} \theta_{x}(0)}{d x^{2}}=0$ at $x=0$ (where $M_{\omega}$ is the bimoment [18]). Thus, the following constants for Eq. (3) are determined:

$$
\begin{gathered}
C_{1}=\frac{T_{x}}{\alpha G J_{x}}\left(\alpha l-\frac{1}{\cosh \alpha l}\right), \quad C_{2}=\frac{T_{x}}{G J_{x}}, \\
C_{3}=0, \quad C_{4}=-\frac{T_{x}}{\alpha G J_{x}}\left(\frac{1}{\cosh \alpha l}\right) .
\end{gathered}
$$

Therefore, the twisting angle of the first case is

$\theta_{x 11}=\frac{T_{x}}{\alpha G J_{x}}\left(\alpha x+\alpha l-\frac{1}{\cosh \alpha l} \sinh \alpha x-\frac{1}{\cosh \alpha l}\right)$.

In the second case, given the assumptions that free warping occurs at both ends $(x=-l$ and $x=0)$, we have $\theta_{x}(0)=0$ and $M_{\omega}=0$ or $\frac{d^{2} \theta_{x}}{d x^{2}}=0$ at $x=0$; and $M_{\omega}=0$ or $\frac{d^{2} \theta_{x}}{d x^{2}}=0$ at $x=-l$. The constants are determined in this case as follows:

$$
C_{1}=0, \quad C_{2}=\frac{T_{x}}{G J_{x}}, \quad C_{3}=0, \quad C_{4}=0 .
$$

Thus, the twisting angle of the second case is

$$
\theta_{x 12}=\frac{T_{x}}{\alpha G J_{x}}(\alpha x) .
$$

In the studied flexure, the joint between elements 1 and 2 was considered to be a flexible joint, which means it was not completely free to warp, but was also not fully restrained. Hence, the joint is partially restrained. A warping restraint factor $K_{1}$ was introduced to find the degree of partial restraint from warping [24]. Then, the warping restraint factor $K_{1}$ was introduced into Eqs. (4) and (5):

$$
\begin{aligned}
\theta_{x 1} & =\frac{T_{x}}{\alpha G J_{x}} \times \\
& \left(\alpha x+\left(1-K_{1}\right)\left(\alpha l-\frac{1}{\cosh \alpha l}(\sinh \alpha x+1)\right)\right) .
\end{aligned}
$$

From Eq. (6), when $K_{1}=0$, the fully-restrained warping occurred at end $x=-l$ (case 1), and free warping occurred when $K_{1}=1$ (case 2). Eq. (6) represents the angle of twist of element 1 with the partial warping constraint at the joint. Therefore, the first- and second-order derivative equations were determined to be:

$$
\theta_{x 1}^{\prime}=\frac{d \theta_{x 1}}{d x}=\frac{T_{x}}{G J_{x}}\left(1+\left(1-K_{1}\right)\left(-\frac{\cosh \alpha x}{\cosh \alpha l}\right)\right),
$$

and

$$
\theta_{x 1}^{\prime \prime}=\frac{d^{2} \theta_{x 1}}{d x^{2}}=\frac{\alpha T_{x}}{G J_{x}}\left(1-K_{1}\right)\left(-\frac{\sinh \alpha x}{\cosh \alpha l}\right) .
$$

The strain energy stored in element 1 is defined as follows [23]:

$$
U_{t 1}=\frac{1}{2} E C_{w} \int_{-l}^{0}\left(\theta_{x 1}^{\prime \prime}\right)^{2} d x+\frac{1}{2} G J_{x} \int_{-l}^{0}\left(\theta_{x 1}^{\prime}\right)^{2} d x .
$$

\subsubsection{Strain Energy of Torsion in Element 2}

$T_{x}$ causes the pure bending in element 2 of the DBLF. The strain energy of bending is defined as follows:

$$
U_{b 2}=\int_{0}^{l} \frac{\left(T_{x}\right)^{2}}{2 E I_{x}} d y,
$$

where $l_{x}=t b_{3} / 12$ is the moment of inertia about the $x$-axis. 


\subsubsection{Strain Energy of Torsion in Element 3}

Similar to element 1 , the joint between elements 2 and 3 of the DBLF is partially restrained from warping. Therefore, at element 3 , the partial warping constraint will occur at $x=-l$ and the full warping constraint will occur at $x=0$. To find the angle of twist of element 3 with the partial warping constraint at the joint, the following two extreme BCs are defined and combined as follows:

First case: we assumed that the full warping constraint occurs at both $x=0$, and $x=-l$. Thus, the BCs are $\theta_{x}(0)=0$ and $\frac{d \theta_{x}(0)}{d x}=0$ at $x=0$; and $\theta_{x}(-l)=0$ and $\frac{d \theta_{x}(-l)}{d x}=0$ at $x=-l$.

The constants for Eq. (3) are determined as follows:

$$
\begin{aligned}
& C_{1}=-\frac{T_{x}}{\alpha G J_{x}}(1-\cosh \alpha l) \frac{1}{\sinh \alpha l}, \quad C_{2}=\frac{T_{x}}{G J_{x}}, \\
& C_{3}=\frac{T_{x}}{\alpha G J_{x}}(1-\cosh \alpha l) \frac{1}{\sinh \alpha l}, \quad C_{4}=-\frac{T_{x}}{\alpha G J_{x}} .
\end{aligned}
$$

Therefore, the twisting angle is:

$$
\begin{aligned}
\theta_{x 31} & =\frac{T_{x}}{\alpha G J_{x}}(\alpha x-\sinh \alpha x+ \\
& \left.\left(\frac{1-\cosh \alpha l}{\sinh \alpha l}\right) \cosh \alpha x-\left(\frac{1-\cosh \alpha l}{\sinh \alpha l}\right)\right) .
\end{aligned}
$$

Second case: we assumed that the full warping constraint occurs at $x=0$, and the free warping constraint occurs at $x=-l$. Thus, the BCs are $\theta_{x}(0)=0$ and $\frac{d \theta_{x}(0)}{d x}=0$ at $x=0, M_{\omega}=0$ or $\frac{d^{2} \theta_{x}(-l)}{d x^{2}}=0$ at $x=-l$.

The constants for Eq. (3) are determined as follows:

$$
\begin{gathered}
C_{1}=\frac{T_{x}}{\alpha G J_{x}} \frac{\sinh \alpha l}{\cosh \alpha l}, \quad C_{2}=\frac{T_{x}}{G J_{x}}, \\
C_{3}=-\frac{T_{x}}{\alpha G J_{x}} \frac{\sinh \alpha l}{\cosh \alpha l}, \quad C_{4}=-\frac{T_{x}}{\alpha G J_{x}} .
\end{gathered}
$$

Thus, the twisting angle is

$$
\begin{aligned}
\theta_{x 32}= & \frac{T_{x}}{\alpha G J_{x}} \times \\
& (\alpha x-\sinh \alpha x-\tanh \alpha l \cosh \alpha x+\tanh \alpha l) .
\end{aligned}
$$

The warping restraint factor $K_{2}$ was introduced into Eqs. (11) and (12):

$$
\begin{aligned}
& \theta_{x 3}=\frac{T_{x}}{\alpha G J_{x}} \times(\alpha x-\sinh \alpha x- \\
& \left(K_{2} \tanh \alpha l+\left(1-K_{2}\right)\left(\frac{1-\cosh \alpha l}{\sinh \alpha l}\right)\right) \times \\
& \cosh \alpha x+K_{2} \tanh \alpha l-\left(1-K_{2}\right)\left(\frac{1-\cosh \alpha l}{\sinh \alpha l}\right),
\end{aligned}
$$

or

$$
\begin{array}{r}
\theta_{x 3}=\frac{T_{x}}{\alpha G J_{x}} \times\left(\alpha x-\sinh \alpha x-K_{t} \cosh \alpha x+\right. \\
\left.K_{2} \tanh \alpha l-\left(1-K_{2}\right)\left(\frac{1-\cosh \alpha l}{\sinh \alpha l}\right)\right),
\end{array}
$$

where $K_{t}=K_{2} \tanh \alpha l+\left(1-K_{2}\right)\left(\frac{1-\cosh \alpha l}{\sinh \alpha l}\right)$.

When $K_{2}=0$, the fully-restrained warping occurs at end $x=-l$ (case 1), and free warping occurs when $K_{2}=1$ (case 2). Eqs. (13) and (14) represent the angle of twist of element 3 with the partial warping constraint at the joint. Therefore, first- and secondorder derivative equations were determined to be:

$$
\theta_{x 3}^{\prime}=\frac{d \theta_{x 3}}{d x}=\frac{T_{x}}{G J_{x}}\left(1-\cosh \alpha x-K_{t} \sinh \alpha x\right)
$$

and

$$
\theta_{x 3}^{\prime \prime}=\frac{d^{2} \theta_{x 3}}{d x^{2}}=\frac{\alpha T_{x}}{G J_{x}}\left(-\sinh \alpha x-K_{t} \cosh \alpha x\right) .
$$

The strain energy stored in element 3 is defined by Eq. [20]:

$$
U_{t 3}=\frac{1}{2} E C_{w} \int_{0}^{l}\left(\theta_{x 3}^{\prime \prime}\right)^{2} d x+\frac{1}{2} G J_{x} \int_{0}^{l}\left(\theta_{x 3}^{\prime}\right)^{2} d x
$$

The torsion constant $J_{x}$ of the rectangular cross section bar was determined by using Eq. (161) published at [19]:

$$
J_{x}=\frac{b t^{3}}{3}\left[1-0.63 \frac{t}{b}\left(1-\frac{t^{4}}{12 b^{4}}\right)\right] .
$$

The warping constant $C_{w}$ with respect to the shear centre is defined [18] as the warping moment of inertia and is calculated as follows:

$$
C_{w}=\int \omega^{2} d A=\frac{(b t)^{3}}{144},
$$

where $\omega$ is the warping function.

From Eqs. (9), (10), and (17), the total strain energy was determined as follows: 


$$
\begin{aligned}
U= & U_{t 1}+U_{b 2}+U_{t 3}=\frac{1}{2} E C_{w} \int_{-l}^{0}\left(\theta_{x 1}^{\prime \prime}\right)^{2} d x+ \\
& \frac{1}{2} G J_{x} \int_{-l}^{0}\left(\theta_{x 1}^{\prime}\right)^{2} d x+\int_{0}^{l} \frac{\left(T_{x}\right)^{2}}{2 E I_{x}} d y+ \\
& \frac{1}{2} E C_{w} \int_{0}^{l}\left(\theta_{x 3}^{\prime \prime}\right)^{2} d x+\frac{1}{2} G J_{x} \int_{0}^{l}\left(\theta_{x 3}^{\prime}\right)^{2} d x .
\end{aligned}
$$

\subsection{Twist Angle}

Integrating each of the terms in Eq. (20) yields:

$$
\begin{aligned}
U= & \frac{T_{x}^{2} l}{2 G J_{x}}+\frac{\alpha E C_{w} T_{x}^{2}\left(K_{1}^{2} \sinh \alpha l-\sinh \alpha l\right)}{2 G^{2} J_{x}^{2} \cosh \alpha l}+\frac{T_{x}^{2} l}{2 E I_{x}}+\frac{T_{x}^{2} l}{2 G J_{x}} \\
& -\frac{\alpha E C_{w} T_{x}^{2}\left(K_{2}^{2} \sinh \alpha l-K_{2}^{2} \sinh \alpha l \cosh \alpha l+\sinh 2 \alpha l\right)}{2 G^{2} J_{x}^{2} \cosh \alpha l(\cosh \alpha l+1)} .
\end{aligned}
$$

Therefore, from Eq. (21), the angle twist at the free end of the DBLF under torsion load $T_{x}$ is obtained as follows:

$$
\begin{aligned}
\theta_{x}= & \frac{\partial U}{\partial T_{x}}=\frac{T_{x} l}{G J_{x}}+\frac{\alpha E C_{w} T_{x}\left(K_{1}^{2} \sinh \alpha l-\sinh \alpha l\right)}{G^{2} J_{x}^{2} \cosh \alpha l}+\frac{T_{x} l}{E I_{x}}+\frac{T_{x} l}{G J_{x}}- \\
& \frac{\alpha E C_{w} T_{x}\left(K_{2}^{2} \sinh \alpha l-K_{2}^{2} \sinh \alpha l \cosh \alpha l+\sinh 2 \alpha l\right)}{G^{2} J_{x}^{2} \cosh \alpha l(\cosh \alpha l+1)} .
\end{aligned}
$$

Eq. (22) includes the partial warping constraints at the joints between elements 1 and 2, and elements 2 and 3.

\section{COMPARISON OF RESULTS}

To verify the results of the theoretical analysis, FEA was conducted by using ANSYS 14.0 commercial FEA software. The parameters of the DBLF with length $l=10 \mathrm{~mm}$, width $b=4 \mathrm{~mm}$, and thickness $t=0.5$ $\mathrm{mm}$ were selected as the default values. The material used in this simulation is aluminium 6061. The torsion moment is $T_{x}=1 \mathrm{~N} \mathrm{~mm}$. The FEA model has a mesh size of $0.1 \mathrm{~mm}$. The sensitive parameters were used to check the agreement between the FEA results and theory results, with the following variations: length $l=5 \mathrm{~mm}$ to $20 \mathrm{~mm}$, width $b=2 \mathrm{~mm}$ to $8 \mathrm{~mm}$, and thickness $t=0.25 \mathrm{~mm}$ to $1 \mathrm{~mm}$. The angles of twist of the DBLF from the theory were numerically calculated and compared using the FEA results with the various factors $K_{1}$ and $K_{2}$. The factors with lower errors compared to the FEA results were chosen and used in the subsequent design steps.

\subsection{Angle of Twist at Default Values}

The rotational displacement of DBLF under torsion load $T_{x}$ is defined by Eq. (22) according to the theoretical analysis. Both warping restraint factor values were chosen at $K_{1}=K_{2}=K=0,0.5$, and 1 . The FEA simulation was also conducted at the default values of flexure. Table 1 shows the results obtained using the theoretical method and FEA. The error for $K=0.5$ was the lowest at $1.3 \%$, and the errors for $K=0$ and $K=1$ were $1.59 \%$ and $8.7 \%$, respectively. The free warping was considered at two joints $(K=1)$ and showed significantly higher errors. The case of $K=0$ with fully-restrained warping at the joints also gave larger errors than in the case of $K=0.5$. This indicates that the results based on the theoretical equation with $K=0.5$ are in good agreement with the FEA results at the default values. Moreover, the two joints between elements 1 and 2 , and 2 and 3 , of the DBLF were well understood with the partial warping constraints $(K=0.5)$. However, to ensure the reliability of the calculations, the sensitive parameters with changes of length, thickness, and width of the DBLF were analysed and tested.

Table 1. Comparison between theory and FEA results at the default values of the DBLF

\begin{tabular}{llcc}
\hline Method & \multicolumn{1}{c}{$\begin{array}{c}\text { Rotational } \\
\text { displacement } \\
\theta_{x}[\mathrm{mrad}]\end{array}$} & $\begin{array}{c}\text { Error between } \\
\text { FEA and } \\
\text { theory [\%] }\end{array}$ \\
\hline FEA & 4.32 & - \\
\hline \multirow{2}{*}{ Theory } & $\begin{array}{l}\text { Fully-restrained warping } \\
(K=0)\end{array}$ & 4.25 & -1.59 \\
\cline { 2 - 4 } & $\begin{array}{l}\text { Partially-restrained } \\
\text { warping }(K=0.5)\end{array}$ & 4.37 & 1.3 \\
\cline { 2 - 4 } & Free warping $(K=1)$ & 4.73 & 8.7 \\
\hline
\end{tabular}

\subsection{Sensitive Parameter Analysis}

Fig. 3 shows the results of $T_{x}$ sensitivity for length $l$ that were obtained by using theory and FEA to simulate the variation of rotation $\theta_{x}$ for lengths $l=5$ $\mathrm{mm}$ to $20 \mathrm{~mm}$ under torsion $T_{x}$ with warping restraint factor values of $K_{1}=K_{2}=K=0,0.5$, and 1 . The values of $\theta_{x}$ are shown in the left $y$-axis and the errors are shown on the right $y$-axis. When length $l$ increases, the rotation $\theta_{x}$ increases linearly in both methods, and the differences in these values are small. However, it is easy to observe the larger differences in the smaller length as shown in Fig. 3 and the values shown in Table 2. The errors from theory $(K=0.5)$ are below $5 \%$, while the errors for $K=0$ and $K=1$ are nearly 


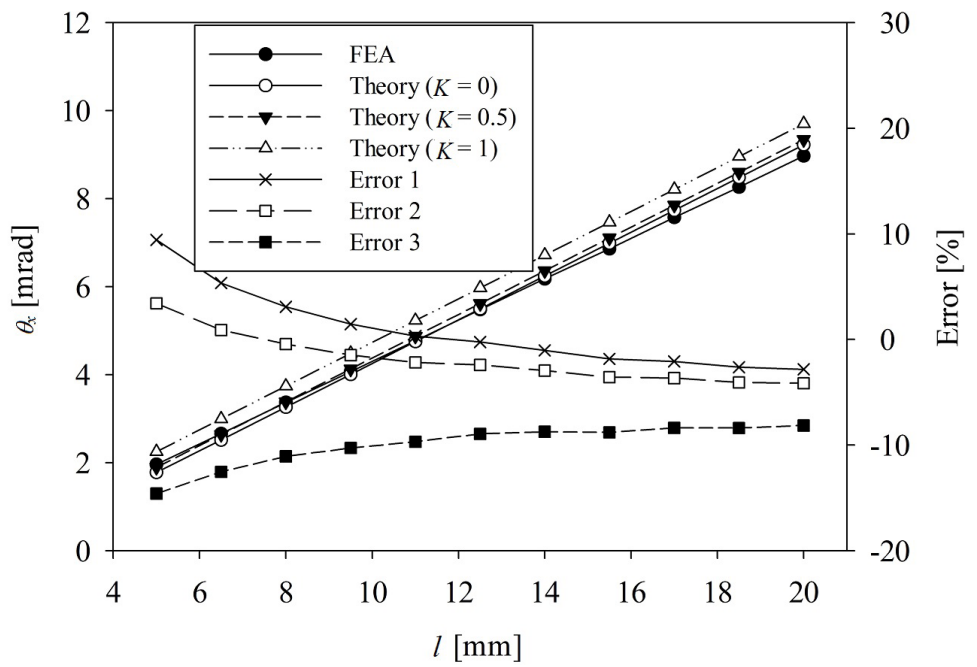

Fig. 3. Variation of $\theta_{x}$ according to length $l$ under torsion $T_{x}$

Table 2. Comparison between theory and FEA

\begin{tabular}{cccccccc}
\hline \multirow{2}{*}{ Length $[\mathrm{mm}]$} & FEA & \multicolumn{3}{c}{ Theory } & \multicolumn{3}{c}{ Error [\%] } \\
\cline { 3 - 8 } & & $K=0$ & $K=0.5$ & $K=1$ & $K=0$ & $K=0.5$ & $K=1$ \\
\hline 5 & 1.960 & 1.775 & 1.893 & 2.247 & 9.44 & 3.42 & -14.61 \\
\hline 6.5 & 2.659 & 2.516 & 2.635 & 2.992 & 5.35 & 0.88 & -12.54 \\
\hline 8 & 3.365 & 3.261 & 3.380 & 3.737 & 3.09 & -0.45 & -11.07 \\
\hline 9.5 & 4.065 & 4.006 & 4.125 & 4.483 & 1.44 & -1.49 & -10.28 \\
\hline 11 & 4.767 & 4.752 & 4.871 & 5.228 & 0.31 & -2.19 & -9.69 \\
\hline 12.5 & 5.483 & 5.497 & 5.616 & 5.974 & -0.25 & -2.43 & -8.95 \\
\hline 14 & 6.179 & 6.243 & 6.362 & 6.719 & -1.04 & -2.96 & -8.75 \\
\hline 15.5 & 6.861 & 6.988 & 7.107 & 7.465 & -1.85 & -3.59 & -8.80 \\
\hline 17 & 7.575 & 7.734 & 7.853 & 8.210 & -2.09 & -3.66 & -8.38 \\
\hline 18.5 & 8.262 & 8.479 & 8.598 & 8.956 & -2.63 & -4.07 & -8.40 \\
\hline 20 & 8.970 & 9.224 & 9.344 & 9.701 & -2.84 & -4.16 & -8.15 \\
\hline
\end{tabular}

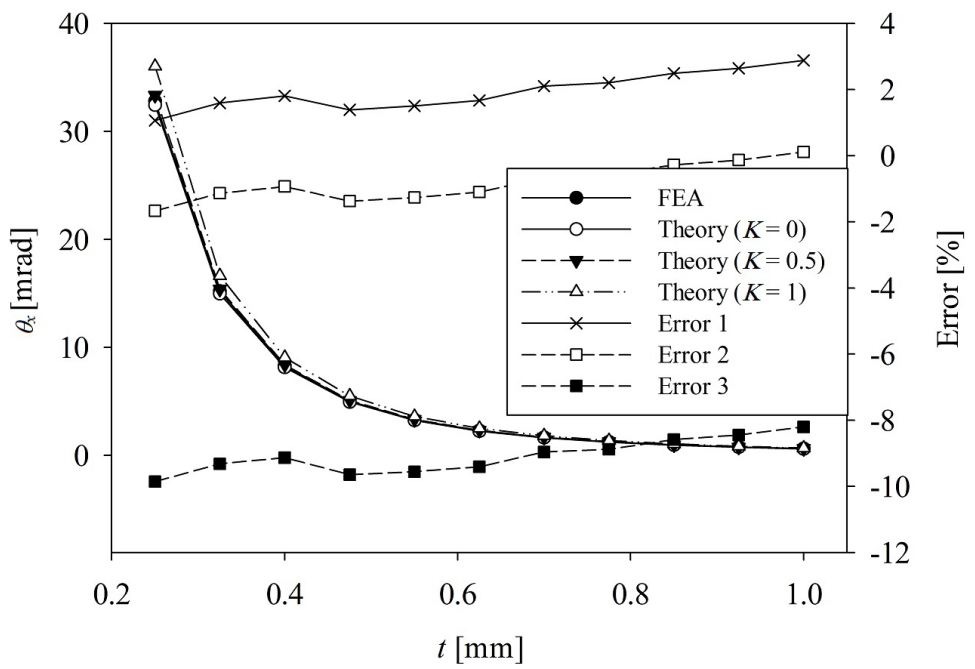

Fig. 4. Variation of $\theta_{x}$ according to thickness $t$ under torsion $T_{x}$ 
Table 3. Rotation $\theta_{x}[\mathrm{mrad}]$ results for theory and FEA according to thickness $t$ under torsion $T_{x}$

\begin{tabular}{cccccccc}
\hline \multirow{2}{*}{ Thickness [mm] } & \multirow{2}{*}{ FEA } & \multicolumn{3}{c}{ Theory } & \multicolumn{3}{c}{ Error [\%] } \\
\cline { 2 - 8 } & & $K=0$ & $K=0.5$ & $K=1$ & $K=0$ & $K=0.5$ & $K=1$ \\
\hline 0.25 & 32.797 & 32.447 & 33.343 & 36.029 & 1.07 & -1.66 & -9.85 \\
\hline 0.325 & 15.214 & 14.971 & 15.386 & 16.632 & 1.60 & -1.13 & -9.32 \\
\hline 0.4 & 8.295 & 8.145 & 8.372 & 9.053 & 1.81 & -0.93 & -9.13 \\
\hline 0.475 & 5.007 & 4.937 & 5.075 & 5.490 & 1.39 & -1.37 & -9.65 \\
\hline 0.55 & 3.279 & 3.230 & 3.321 & 3.593 & 1.50 & -1.26 & -9.56 \\
\hline 0.625 & 2.275 & 2.237 & 2.300 & 2.489 & 1.67 & -1.10 & -9.41 \\
\hline 0.7 & 1.654 & 1.619 & 1.665 & 1.802 & 2.10 & -0.66 & -8.96 \\
\hline 0.775 & 1.241 & 1.214 & 1.248 & 1.351 & 2.21 & -0.57 & -8.88 \\
\hline 0.85 & 0.960 & 0.937 & 0.963 & 1.043 & 2.49 & -0.28 & -8.59 \\
\hline 0.925 & 0.760 & 0.740 & 0.761 & 0.824 & 2.64 & -0.13 & -8.45 \\
\hline 1 & 0.615 & 0.597 & 0.614 & 0.665 & 2.88 & 0.11 & -8.20 \\
\hline
\end{tabular}

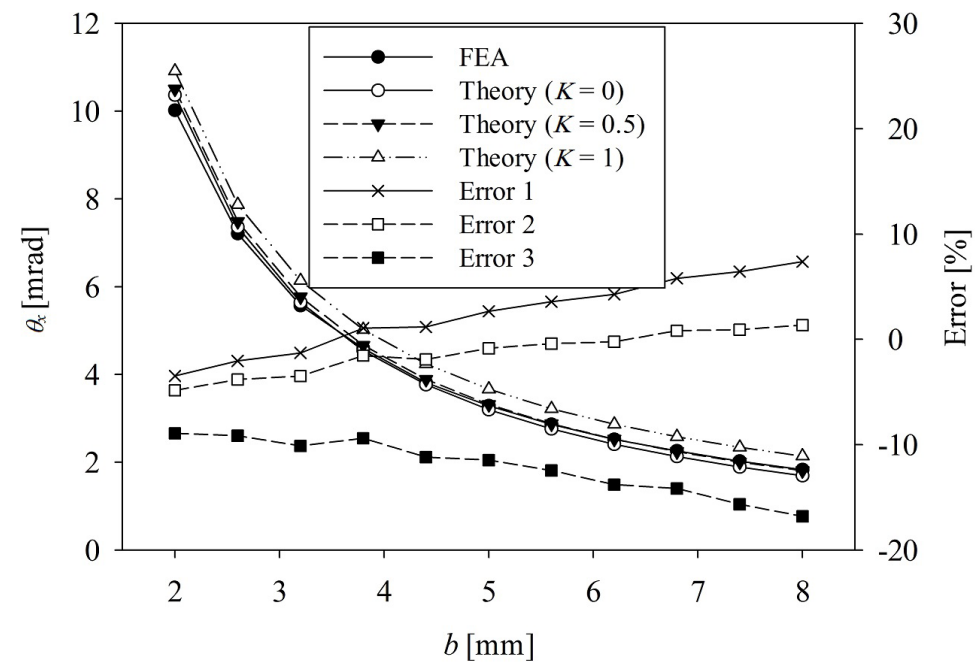

Fig. 5. Variation of $\theta_{x}$ according to width $b$ under torsion $T_{x}$

$10 \%$ and $15 \%$, respectively. Thus, the theoretical result with $K=0.5$ is in better agreement with the FEA results.

Similarly, Fig. 4 shows the results of $T_{x}$ sensitivity for thickness $t$ with the variation of thickness $t=0.25$ $\mathrm{mm}$ to $1 \mathrm{~mm}$. When thickness $t$ increases, the rotation $\theta_{x}$ decreases nonlinearly for both methods, and the curves of these values are quite similar. However, there are large differences in the comparison of errors with FEA, as shown in Fig. 4, and the values shown in Table 3. In this simulation, the errors of theory $(K=0.5)$ are below $2 \%$, while the errors for $K=0$ and $K=1$ are nearly $3 \%$ and $10 \%$, respectively. These results show that the theoretical result with $K=0.5$ is the most accurate prediction.

Fig. 5 shows the results of the variation of rotation $\theta_{x}$ according to the width $b=2 \mathrm{~mm}$ to 8 mm under torsion $T_{x}$ based on theory and FEA. The warping restraint factors values are the same as in the aforementioned two cases. When width $b$ increases, the rotation $\theta_{x}$ is decreasing in both methods. The errors compared with FEA are presented in Fig. 5 and Table 4 . The errors of theory $(K=0.5)$ are below $5 \%$ while the errors for $K=0$ and $K=1$ are up to nearly $8 \%$ and $17 \%$, respectively. These graphs also reveal that the results of theory with $K=0.5$ are in strong agreement with the results of FEA.

In summary, the simulation was conducted at the default values and for variations of length, thickness, and width values and compared with the numerical calculations from the theoretical equations. The warping restraint factors values were varied as follows: $K_{1}=K_{2}=K=0,0.5$, and 1 , corresponding to fixed (no warping), partially restrained warping, and free (free warping), respectively, in the theoretical method. The results are presented in Figs. 3 to 5 and 
Table 4. Rotation $\theta_{x}[\mathrm{mrad}]$ from theory and FEA according to width $b$ under torsion $T_{x}$

\begin{tabular}{cccccccc}
\hline \multirow{2}{*}{ Width [mm] } & \multirow{2}{*}{ FEA } & \multicolumn{3}{c}{ Theory } & \multicolumn{3}{c}{ Error [\%] } \\
\cline { 2 - 8 } & & $\mathrm{K}=0$ & $\mathrm{~K}=0.5$ & $\mathrm{~K}=1$ & $\mathrm{~K}=0$ & $\mathrm{~K}=0.5$ & $\mathrm{~K}=1$ \\
\hline 2 & 10.018 & 10.367 & 10.504 & 10.913 & -3.49 & -4.85 & -8.93 \\
\hline 2.6 & 7.210 & 7.358 & 7.486 & 7.869 & -2.05 & -3.83 & -9.15 \\
\hline 3.2 & 5.574 & 5.646 & 5.769 & 6.138 & -1.29 & -3.50 & -10.13 \\
\hline 3.8 & 4.590 & 4.541 & 4.661 & 5.021 & 1.06 & -1.55 & -9.40 \\
\hline 4.4 & 3.813 & 3.768 & 3.886 & 4.239 & 1.18 & -1.91 & -11.18 \\
\hline 5 & 3.285 & 3.197 & 3.313 & 3.662 & 2.68 & -0.86 & -11.47 \\
\hline 5.6 & 2.861 & 2.758 & 2.873 & 3.217 & 3.59 & -0.42 & -12.46 \\
\hline 6.2 & 2.517 & 2.410 & 2.523 & 2.865 & 4.27 & -0.24 & -13.79 \\
\hline 6.8 & 2.258 & 2.127 & 2.240 & 2.578 & 5.80 & 0.81 & -14.16 \\
\hline 7.4 & 2.023 & 1.893 & 2.005 & 2.340 & 6.43 & 0.91 & -15.66 \\
\hline 8 & 1.832 & 1.697 & 1.808 & 2.140 & 7.39 & 1.34 & -16.80 \\
\hline
\end{tabular}

Tables 1 to 4 . The errors in the no warping case $(K=0)$ sensitivity analysis are up to $10 \%$. The errors in the free warping case $(K=1)$ are up to $17 \%$. The errors in the partially-restrained warping case $(K=0.5)$ are lower than $5 \%$. These results indicate that the partially-restrained warping at the two joints of DBLF is well explained with factors $K_{1}=K_{2}=K=0.5$.

\section{CONCLUSION}

We analysed the angle of twist of the DBLF under torsion with consideration of the partially-restrained warping at two joints and the warping restraint factor $K$. The strain energy of each element is derived, and the total strain energy of the DBLF is presented based on Castigliano's theorem. The equations of rotational displacement of the DBLF under torsion are presented. The finite element simulation was conducted at the default values, and for variations of length, thickness, and width, using various values of the warping restraint factors. Our results indicate that the theoretical equations with consideration of the double partially-restrained warping effects $\left(K_{1}=K_{2}=K=0.5\right)$ for the DBLF can provide a sufficiently accurate prediction of torsional angle in the design of precision machines.

\section{ACKNOWLEDGEMENTS}

This work was supported by the Business for University Entrepreneurship Center, funded by the Korea Small and Medium Business Administration in 2015 2016. The authors gratefully acknowledge this support.

\section{REFERENCES}

[1] Brouwer, D.M., Meijaard, J.P., Jonker, J.B. (2013). Large deflection stiffness analysis of parallel prismatic leaf-spring flexures. Precision Engineering, vol. 37, no. 3, p. 505-521, D0l:10.1016/j.precisioneng.2012.11.008.

[2] Hayashi, M., Fukuda, M. (2012). Generation of nanometer displacement using reduction mechanism consisting of torsional leaf spring hinges. International Journal of Precision Engineering and Manufacturing, vol. 13, no. 5, p. 679-684, Dol:10.1007/s12541-012-0088-1.

[3] Yong, Y.K., Aphale, S.S., Moheimani, S.O.R. (2009). Design, identification, and control of a flexure-based $X Y$ stage for fast nanoscale positioning. IEEE Transactions on Nanotechnology, vol. 8, no. 1, p. 46-54, Dol:10.1109/TNAN0.2008.2005829.

[4] Kim, J.-J., Choi, Y.-M., Ahn, D., Hwang, B., Gweon, D.-G., Jeong, J. (2012). A millimeter-range flexure-based nano-positioning stage using a self-guided displacement amplification mechanism. Mechanism and Machine Theory, vol. 50, p. 109120, D0I:10.1016/j.mechmachtheory.2011.11.012.

[5] Fukada, S., Nishimura, K. (2007). Nanometric positioning over a one-millimeter stroke using a flexure guide and electromagnetic linear motor. International Journal of Precision Engineering and Manufacturing, vol. 8, no. 2, p. 4953.

[6] Xu, Q.S. (2014). Design and development of a compact flexure-based $X Y$ precision positioning system with centimeter range. IEEE Transactions on Industrial Electronics, vol. 61, no. 2, p. 893-903, DOl:10.1109/TIE.2013.2257139.

[7] Koseki, Y., Tanikawa, T., Koyachi, N., Arai, T. (2000). Kinematic analysis of translational 3-DOF micro parallel mechanism using matrix method. Proceedings of International Conference on Intelligent Robots and Systems, vol. 1, p. 786-792, DOI:10.1109/IROS.2000.894700.

[8] Kim, J.H., Kim, S.H., Kwak, Y. K. (2004). Development and optimization of 3-D bridge-type hinge mechanisms. Sensors and Actuators A: Physical, vol. 116, no. 3, p. 530-538, DOI:10.1016/j.sna.2004.05.027.

[9] Ryu, J.W., Gweon, D.-G., Moon, K.S. (1997). Optimal design of a flexure hinge based XY $\varphi$ wafer stage. Precision Engineering, 
vol. 21, no. 1, p. 18-28, Dol:10.1016/S0141-6359(97)000640.

[10] Li, Y.M., Xu, Q.S. (2011). A novel piezoactuated XY stage with parallel, decoupled, and stacked flexure structure for micro-/ nanopositioning. IEEE Transactions on Industrial Electronics, vol. 58, no. 8, p. 3601-3615, DOl:10.1109/TIE.2010.2084972.

[11] Lobontiu, N. (2014). Compliance-based matrix method for modeling the quasi-static response of planar serial flexurehinge mechanisms. Precision Engineering, vol. 38, no. 3, p. 639-650, D0I:10.1016/j.precisioneng.2014.02.014.

[12] Park, E.J., Shim, J.S., Lee, D.Y., Lee, J.W. (2010). A Double-bent Planar Leaf Flexure Guide for a Nano-scanner. Journal of the Korean Physical Society, vol. 57, no. 6, p. 1581-1588.

[13] Nguyen, H.N., Lee, D.Y. (2015). Bending analysis of a single leaf flexure using higher-order beam theory. Structural Engineering and Mechanics, vol. 53, no. 4, p. 781-790, DOI:10.12989/sem.2015.53.4.781.

[14] Kim, K., Ahn, D., Gweon, D. (2012). Optimal design of a 1-rotational DOF flexure joint for a 3-DOF H-type stage. Mechatronics, vol. 22, no. 1, p. 24-32, D0l:10.1016/j. mechatronics.2011.10.002.

[15] Nguyen, N.H., Lim, B.D., Lee, D.Y. (2015). Displacement analysis of a single-bent leaf flexure under transverse load. International Journal of Precision Engineering and Manufacturing, vol. 16, no. 4, p. 749-754, Dol:10.1007/ s12541-015-0099-9.

[16] Bhagat, U., Shirinzadeh, B., Clark, L., Chea, P., Qin, Y., Tian, Y., Zhang, D. (2014). Design and analysis of a novel flexure-based 3-DOF mechanism. Mechanism and Machine Theory, vol. 74, p. 173-187, D0l:10.1016/j.mechmachtheory.2013.12.006.
[17] Lobontiu, N., Garcia, E. (2005). Mechanics of Microelectromechanical Systems, Kluwer, New York, Dol:10.1007/b100026.

[18] Pilkey, W.D. (2002). Analysis and Design of Elastic Beams: Computational Methods. John Wiley \& Sons, Inc., p. 323-329, New York, DOl:10.1002/9780470172667.

[19] Timoshenko, S.P., Goodier, J.N. (1970). Theory of Elasticity. McGraw-Hill, New York.

[20] Bishplinghoff, R.L., Ashley, H., Halfman, R.L. (1996). Aeroelasticity. Dover Publications, Inc., New York.

[21] Sapountzakis, J.E. (2013). Bars under torsional loading: A generalized beam theory approach", ISRN Civil Engineering, vol. 2013, p. 1-39, Dol:10.1155/2013/916581.

[22] Erkmen, R.E., Mohareb, M. (2006). Torsion analysis of thinwalled beams including shear deformation effects. Thin-Walled Structures, vol. 44, no. 10, p. 1096-1108, D0l:10.1016/j. tws.2006.10.012.

[23] Kujawa M. (2011). Torsion of restrained thin-walled bar of open constant bisymmetric cross-section. Task Quarterly, vol. 16, no. 1, p. 5-15.

[24] Al-HaKeem, A.H. (1991). Structural Analysis of Truck Chassis Frames under Longitudinal Loads Considering Bimoment Effects. Ph.D. thesis, Cranfield Institute of Technology, Cranfield.

[25] Yang, Y.B., McGuire, W. (1984). A procedure for analysing space frames with partial warping restraint. International Journal for Numerical Methods in Engineering, vol. 20, no. 8, p. 1377-1398, DOl:10.1002/nme.1620200803.

[26] Nghia, N.H., Lim, B.D., Lee, D.Y. (2015). Torsional analysis of a single-bent leaf flexure. Structural Engineering and Mechanics, vol. 54, no. 1, p. 189-198, D0l:10.12989/ sem.2015.54.1.189. 